OPEN ACCESS

Edited by:

Xiaogan $\mathrm{Li}$,

Dalian University of Technology

(DUT), China

Reviewed by:

Zhanying Zhang

Henan Polytechnic University, China

Fengmin Liu,

Jilin University, China

*Correspondence:

Xiaotao Zu

xtzu@uestc.edu.cn

Weilie Zhou

wzhou@uno.edu

†These authors have contributed equally to this work

Specialty section:

This article was submitted to Functional Ceramics,

a section of the journal

Frontiers in Materials

Received: 26 June 2019

Accepted: 26 August 2019

Published: 13 September 2019

Citation:

Liu C, Zheng Z, Chen J, Su H, Xiang X, Zu X and Zhou W (2019)

Metal Decoration and Magnetic Field Effect on the Electrical Breakdown

Voltage for $\mathrm{ZnO}$ Nanorods Gas Ionization Sensor. Front. Mater. 6:220.

doi: 10.3389/fmats.2019.00220

\section{Metal Decoration and Magnetic Field Effect on the Electrical Breakdown Voltage for ZnO Nanorods Gas Ionization Sensor}

\author{
Chunming Liu ${ }^{1,2 t}$, Zhi Zheng ${ }^{1,2 t}$, Jiajun Chen ${ }^{2}$, Haiqiao Su ${ }^{2}$, Xia Xiang ${ }^{1}$, Xiaotao $\mathrm{Zu}^{1 *}$ and \\ Weilie Zhou ${ }^{2 \star}$ \\ ${ }^{1}$ School of Physics, University of Electronic Science and Technology of China, Chengdu, China, ${ }^{2}$ Advanced Materials \\ Research Institute, University of New Orleans, New Orleans, LA, United States
}

$\mathrm{ZnO}$ nanorod arrays with sharp tips were synthesized via chemical vapor deposition and utilized as the anode for gas ionization sensor. The effects of metal ( $\mathrm{Mn}, \mathrm{Ni}_{81} \mathrm{Fe}_{19}$, and $\mathrm{Au}$ ) decoration and magnetic field on the electrical breakdown voltage were studied. Compared with the bare $\mathrm{ZnO}$ nanorod, metal decorated $\mathrm{ZnO}$ nanorod arrays in the magnetic field can effectively reduce the breakdown voltage for the air to $20 \mathrm{~V}$. The possible mechanism is that metal decoration produces small size protuberance on the surface of $\mathrm{ZnO}$ nanorods and then enhances the electrical field near the tips. The magnetic field exerts action on the motion of the electron and therefore increases electron impact ionization efficiency and second electron emission efficiency.

Keywords: gas ionization sensor, $\mathrm{ZnO}$, metal decoration, magnetic field effect, electrical breakdown voltage

\section{INTRODUCTION}

Gas sensors have a significant impact in the areas of environmental emission control, public security, automotive application, workplace hazard monitoring, medical diagnosis (Zhang et al., 2001; Modi et al., 2003; Hou et al., 2006; Li et al., 2010, 2019a,b; Chen et al., 2011; Yao et al., 2012; Wang et al., 2015). Among different gas sensors, gas ionization sensors have shown great promise due to their high selectivity and sensitivity, giving that distinct gases have their unique electrical breakdown voltage (Zhang et al., 2001; Modi et al., 2003). However, traditional gas ionization sensor used in chromatography and mass spectrograph cannot be used on site because of the high-power consumption and risky operation high-voltage (Zhang et al., 2001; Modi et al., 2003; Hou et al., 2006). Thus, decreasing the operation voltage of gas ionization sensors is of great importance in practical applications. Due to high electric fields generated at sharp tips of nanotubes and nanorods electrode, new types of gas ionization sensor using nanomaterials as electrode has been developed (Zhang et al., 2001, 2013; Modi et al., 2003; Hou et al., 2006; Sadeghian and Kahrizi, 2007; Liao et al., 2008; Sadeghian, 2008; Azmoodeh et al., 2009; Baghgar et al., 2009; Huang et al., 2009; Min et al., 2011; Wang et al., 2011). The reported nanomaterials electrode includes various materials, such as carbon nanotubes (Zhang et al., 2001, 2013; Modi et al., 2003; Hou et al., 2006; Baghgar et al., 2009; Huang et al., 2009), ZnO nanorods (Liao et al., 2008; Min et al., 2011; Wang et al., 2011), gold nanorods (Sadeghian and Kahrizi, 2007; Sadeghian, 2008), and silver nanorods (Azmoodeh et al., 2009). With these nanomaterials used as the electrode, the gas electrical breakdown voltage $\left(V_{\mathrm{EB}}\right)$ is realized at a relatively low applied voltage, which is reduced several-folds in comparison to traditional plane electrodes 
(Zhang et al., 2001; Modi et al., 2003; Hou et al., 2006; Sadeghian and Kahrizi, 2007; Liao et al., 2008; Sadeghian, 2008; Azmoodeh et al., 2009; Baghgar et al., 2009; Min et al., 2011; Wang et al., 2011). Moreover, this new type of gas ionization sensor was proved to be reversible and fast as there is no chemical absorption involved.

It was reported that field enhancement is related to the morphology of tips (Pan et al., 2010). Among plane, tapered, and abruptly sharpened tips, the abruptly sharpened tips have the highest electric field enhancement factor (Pan et al., 2010). As a widely studied material, the tip morphology of $\mathrm{ZnO}$ nanorods can be easily adjusted. In addition, it was confirmed that as an electrode material of gas ionization sensor, $\mathrm{ZnO}$ nanorods have better stability and anti-oxidation behavior than carbon nanotubes (Liao et al., 2008). However, the $V_{\mathrm{EB}}$ for $\mathrm{ZnO}$ nanorods electrode is much higher than that for carbon nanotubes electrode. For example, the $V_{\mathrm{EB}}$ of air for $\mathrm{ZnO}$ nanorods electrode is $188 \mathrm{~V}$ higher than that for carbon nanotube electrode (Liao et al., 2008). Although Pd capping and electrode separation narrowing were reported to decrease $V_{\mathrm{EB}}$ of gas (Wang et al., 2011), the $V_{\mathrm{EB}}$ of gas is still much higher than a safe voltage (the safe operation criteria voltage is below $36 \mathrm{~V}$; Hou et al., 2006). For the $\mathrm{ZnO}$ nanorods electrode, the $V_{\mathrm{EB}}$ of air is as high as $335 \mathrm{~V}$ though the electrode separation is reduced to $25 \mu \mathrm{m}$ (Liao et al., 2008). Even for the carbon nanotube electrode, the $V_{\mathrm{EB}}$ of air is still $208 \mathrm{~V}$ when the electrode separation is $25 \mu \mathrm{m}$ (Liao et al., 2008). For the capacitance structured gas-gap device, further decreasing the separation of the electrode is very difficult (Hou et al., 2006). Sole Pd capping can only decrease the $V_{\mathrm{EB}}$ of air from 363 to $341 \mathrm{~V}$ (Wang et al., 2011). It is evidently seen that sole metal capping and electrode separation decreasing are not enough to reduce the $V_{\mathrm{EB}}$ below the safe operation criteria.

The magnetic field effect on the electrical breakdown of gases was widely studied (Haydon et al., 1971; Li and Uhm, 2004; Petraconi et al., 2004; Radjenović and Radjenović, 2006; Wais et al., 2011). An important factor is that both longitudinal (Petraconi et al., 2004) (parallel to the electrical field) and transverse (Li and Uhm, 2004; Radjenović and Radjenović, 2006; Wais et al., 2011) (perpendicular to electrical field) magnetic field are facilitating for gas electrical breakdown for the device with plane parallel electrodes with large separation distance (distance is about several centimeters). This behavior should be beneficial for decreasing $V_{\mathrm{EB}}$ for the device with the nanomaterial electrode since the electrical field direction near nanotips is not uniform. To the best of our knowledge, there is no report on the magnetic field effect on gas ionization for $\mathrm{ZnO}$ nanorods electrode. In this paper, we studied the metal decoration and magnetic field effect on the electrical breakdown voltage for $\mathrm{ZnO}$ nanorods gas ionization sensor. By combining the metal decoration and magnetic field effect, the $V_{\mathrm{EB}}$ of air can be well-reduced to $20 \mathrm{~V}$, therefore provide a promising way to improve the performance for gas ionization sensors.

\section{MATERIALS AND METHODS}

$\mathrm{ZnO}$ nanorods fabrication process is similar to that reported by Xiao et al. (2008). The $\mathrm{ZnO}$ nanorods samples were fabricated using a conventional horizontal tube furnace with a quartz tube.
The substrate is n-type $\mathrm{Si}$ wafers with a $300 \mathrm{~nm}$ thermal oxide $\mathrm{SiO}_{2}$ layer. The crystal orientation of the $\mathrm{Si}$ wafer is (100). The size of the substrate is about $0.05 \times 0.8 \times 1.2 \mathrm{~cm}^{3}$. These substrates were first ultrasonically cleaned in ultrasonic with acetone, IPA, and distilled water and dried by nitrogen flow. After the substrate being carefully cleaned, about $30 \mathrm{~nm}$ thick gold film was sputter deposited using a Cressington 308R thin film coating system. The thickness of the film was monitored by a quartz oscillator equipped in the system. The base pressure of the sputter chamber is about $1 \times 10^{-6} \mathrm{mbar}$, and the working pressure is about 0.02 mbar. Secondly, a drop coating process was used to deposit $\mathrm{ZnO}$ seeds layer on the gold film. Zinc acetate (Fisher scientific) ethanol solution of $0.015 \mathrm{M}$ was prepared. About 20 $\mu l$ of this solution was dropped on to the surface of the gold film. The substrate was then dried by nitrogen flow after waiting for about $30 \mathrm{~s}$. This process was repeated for six cycles. Thirdly, Zn powder (Damon 5918J25) of about 0.5 grams was loaded on the center of an alumina boat and served as the source material. Substrates with gold film and seeds layer were put over the source material about $2-3 \mathrm{~mm}$ above and different distance to the center of the boat. Substrates were loaded with face down. The alumina boat that contained both the source material and the substrates was pushed to the center of the quartz tube, which was then pumped to a pressure of about 8 Torr with a mechanical pump. Argon gas was then introduced into the tube as a carrier gas with a flux of 50 standard cubic centimeters per minute (SCCM). The furnace temperature was subsequently increased from room temperature to $550^{\circ} \mathrm{C}$ within $50 \mathrm{~min}$. During the heating up process, oxygen gas was introduced at a flux of 5 SCCM when the temperature of furnace reached $450^{\circ} \mathrm{C}$. The temperature of the furnace was maintained at $550^{\circ} \mathrm{C}$ for $30 \mathrm{~min}$. After that, the furnace temperature was decreased from 550 to $450^{\circ} \mathrm{C}$ within $40 \mathrm{~min}$, and then the furnace was turned off and the substrates were allowed to cool down naturedly to room temperature. The morphology of the sample was measured with a Zeiss (LEO) 1530VP field emission scanning electron microscope (FESEM) and a JEOL 2010 transmission electron microscope (TEM). The composition analyses were conducted by the X-ray energy dispersive spectroscopy (EDS) equipped on the FESEM and TEM. For TEM observations, $\mathrm{ZnO}$ nanorods were scraped from the substrate by a blade and dispersed in ethanol using an ultra-sonicator. The $\mathrm{ZnO}$ nanorods dispersed in ethanol were introduced dropwise onto a $\mathrm{Cu}$ grid covered with carbon films. The crystal structure of the sample was characterized by Philips X'pert MPD X-ray diffraction (XRD). To fabricate the device, the $\mathrm{Si} / \mathrm{SiO}_{2}$ substrate with $30 \mathrm{~nm}$ gold films was fixed on a glass substrate and used as the cathode. The $\mathrm{ZnO}$ nanorod sample was biased as the anode. The separation between anode and cathode is kept constant as about $200 \mu \mathrm{m}$. The testing organic gases were obtained by putting $15 \mathrm{ml}$ corresponding organic liquid into a flask with a volume of about $250 \mathrm{ml}$. The flask was then sealed with a rubber stopper and kept in room temperature for about $24 \mathrm{~h}$. Then the rubber stopper was taken away and the ionization sensor was put into the atmosphere in the flask. The flask was sealed again with two leads from sensor outside the flask for the testing. The breakdown voltage of air was tested by putting the sensor in the open air condition. The volt-ampere (V-I) character of the device was measured by a Keithley 2400 source 


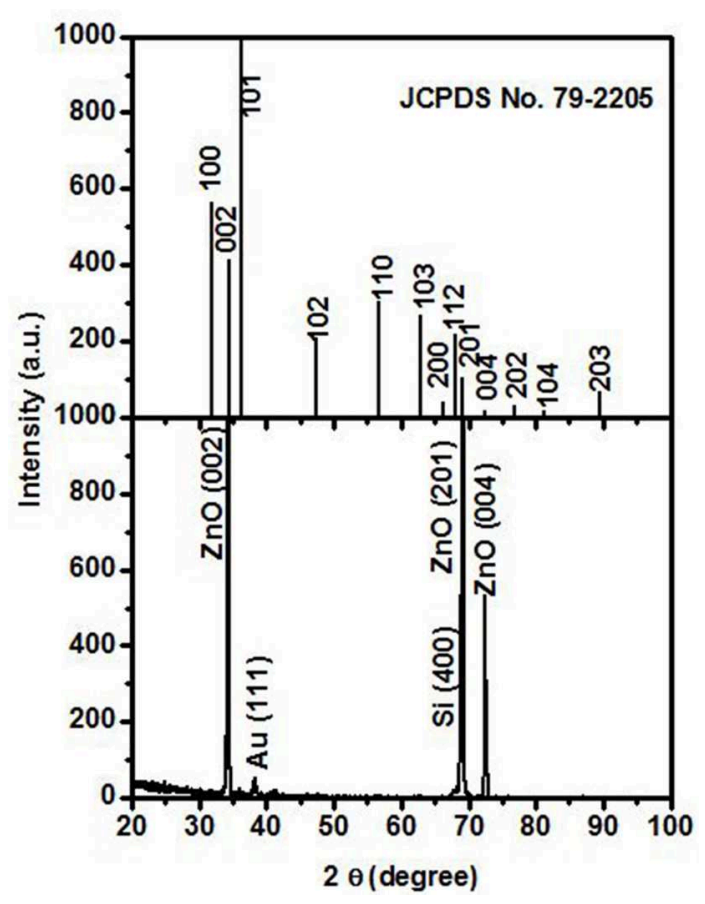

FIGURE 1 | XRD of ZnO nanorods sample. The up panel and the low panel are from JCPDS No. 79-2205 and ZnO nanorods sample, respectively.

meter. The metal decoration was deposited on the $\mathrm{ZnO}$ nanorods sample by sputter deposition using a Cressington 308R thin film coating system. The thickness of decoration was monitored by a quartz oscillator equipped in the system. The magnetic field was produced by a permanent magnet. The magnetic field was measured using Lakeshore 450 Gaussmeter.

\section{RESULTS AND DISCUSSION}

Figure 1 shows the XRD pattern of $\mathrm{ZnO}$ nanorods sample. The up panel of Figure 1 is the XRD pattern of JCPDS No. 792205. The obtained $\mathrm{ZnO}$ nanorods sample has the hexagonal structure as confirmed by XRD measurement. The diffraction peaks located at 34.31 and $72.47^{\circ}$ are indexed, respectively, as (002) and (004) of $\mathrm{ZnO}$ (JCPDS No. 79-2205). The lattice constant $c$ is calculated to be about $0.522 \mathrm{~nm}$. The peaks located at $69.04^{\circ}$ may come from (004) of Si (JCPDS No. 75-0590) and (201) diffraction of $\mathrm{ZnO}$. The peak at about $38.23^{\circ}$ is indexed as (111) diffraction from gold (JCPDS No.04-0784).

The typical morphology of $\mathrm{ZnO}$ nanorods are shown in Figure 2. Figures 2A,B are the low and high magnified $30^{\circ}$ tilt view of FESEM images, respectively. As shown in Figure 2B, the nanorod has a large base and abruptly sharp tips with a diameter of about $40 \mathrm{~nm}$. The formation mechanism of the abruptly sharp tips of $\mathrm{ZnO}$ is not clear at present. It was reported that different tip shapes of $\mathrm{ZnO}$ nanorods can be controlled by the substrate direction relative to the gas flow during fabrication (Pan et al., 2005). The local oxygen concentration around the substrate also plays an important role in determining the tip shapes of $\mathrm{ZnO}$ nanorods (Pan et al., 2005). It was reported that $\mathrm{ZnO}$ nanorods with abruptly sharp tips have an advantage in electrical field enhancement (Pan et al., 2010). The sparse vertically aligned tips are useful to reduce the screening effect of the nearest neighbor tips (Pan et al., 2010). Taking into account the screening effect, the electrical field at the tip can be expressed as Filip et al. (2001):

$$
E_{l o c}=s \frac{V}{r}+(1-s) \frac{V}{d}
$$

where $V$ is the applied voltage between electrodes, $d$ is the cathode to anode distance, $r$ is the emitter tip's radius of curvature, and $s$ is the factor in evaluating the degree of screening effect, which ranges from 0 (for extremely high-density tips arrays) to 1 (for a single tip). The higher is the density of tips, the greater the screening effect. The local field on every tip decreases quickly with the distance between the nearest neighbor tips decreasing (Nilsson et al., 2000). It is seen from Equation (1) that the electrical field has no dependence on the length of the nanorod. However, other literature reported that the electrical field at tips is also dependent on the length of nanorods (Wang et al., 2004). The common in literature is that the smaller the tip diameter, the higher the electrical field near the tip surface (Filip et al., 2001; Wang et al., 2004). This is one reason that small size metal decoration on the surface of nanotips would enhance the electrical field.

The obtained $\mathrm{ZnO}$ nanorods sample was biased as an anode electrode of the device. A typical scheme of the device is shown in Figure 3. A silica substrate with $30 \mathrm{~nm}$ gold film was used as a cathode. It was found that the $\mathrm{ZnO}$ nanorods electrode is able to ionize and distinguish acetone, IPA, and ethanol gas. Figure 4 shows the I-V curves for these gases. As indicated in Figure 4, the current first increases linearly with the voltage increasing and then increases abruptly when the voltage is above a certain threshold. It was reported that the I-V curve of gas ionization sensor obeys the Ohmic law when the electrical field strength is less than the ionization threshold (Sadeghian, 2008). The current is determined by the movement of existing radiationgenerated electron-ion pairs and proportional to electrical filed (Sadeghian, 2008). When the voltage is above the threshold, the current increase sharply because of the gas ionization is initiated (Sadeghian, 2008). The ionization energy of acetone, IPA, ethanol is $9.69,10.12$, and $10.62 \mathrm{eV}$, respectively (Huang et al., 2009). The $V_{\mathrm{EB}}$ is determined to be about $6.9,17.8$, and $19.3 \mathrm{~V}$, respectively, which is distinct for different gases. It is seen that gas with higher ionization energy has a higher $V_{\mathrm{EB}}$. This is reasonable as that $V_{\mathrm{EB}}$ is mainly determined by the ionization energy of gas (Sadeghian and Kahrizi, 2007). However, the device cannot make air electrical breakdown even though the applied voltage reaches $210 \mathrm{~V}$. This may be due to the ionization energy of oxygen and nitrogen is higher than these organic gases. The ionization energy of oxygen and nitrogen is 12.06 and $15.58 \mathrm{eV}$, respectively.

In order to ionize gas with higher ionization energy, metal decoration, and magnetic field are used to ionize air as an example. Figures $\mathbf{5 A - C}$ show the TEM images for pure $\mathrm{ZnO}$ nanorods, $5 \mathrm{~nm} \mathrm{Mn}$ and a $15 \mathrm{~nm}$ Mn decorated $\mathrm{ZnO}$ nanorods, respectively. The size of $\mathrm{Mn}$ particle is about $5 \mathrm{~nm}$. As shown 

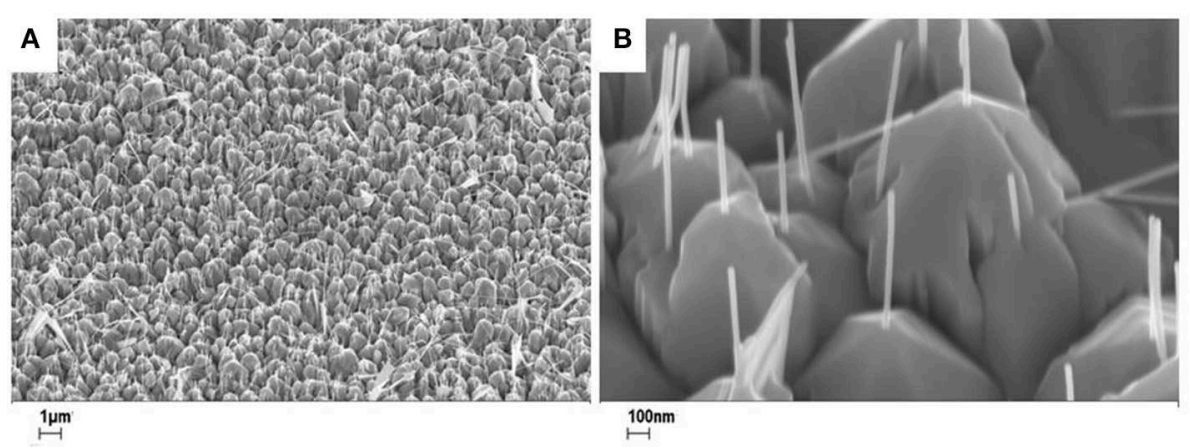

FIGURE 2 | FESEM of ZnO nanorods: lower magnified (A) and higher magnified (B) $30^{\circ}$ tilt view.

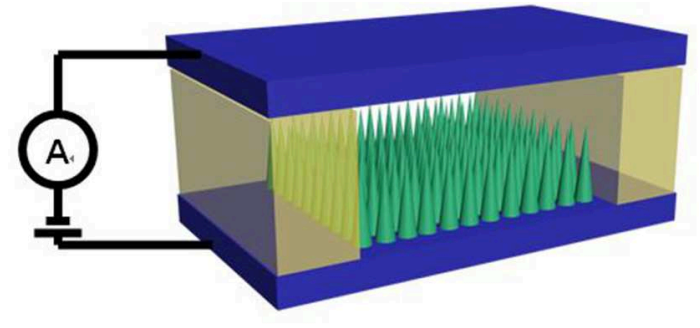

FIGURE 3 | The structural scheme of device. The $\mathrm{ZnO}$ nanorods were biased as anode.

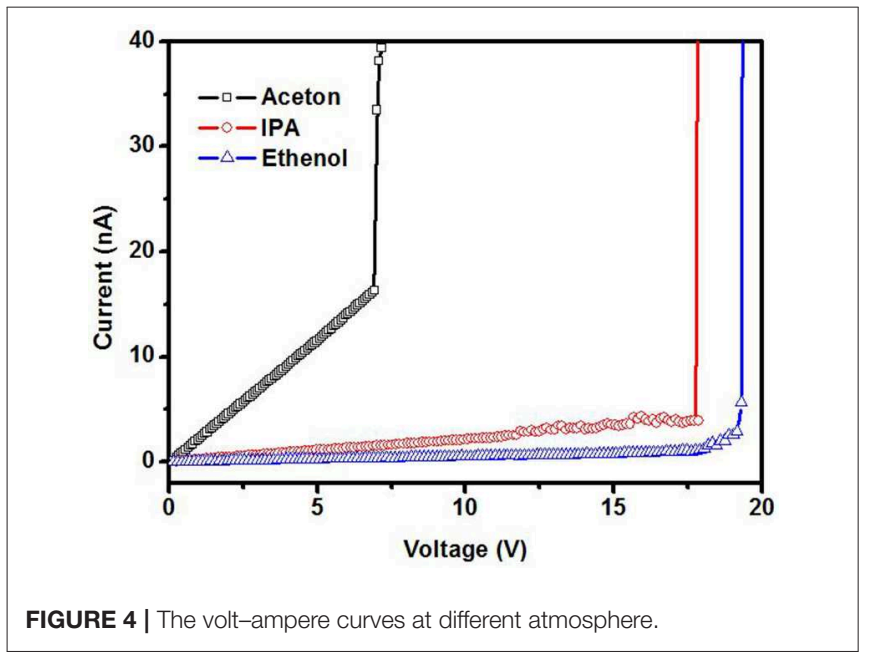

in the TEM images, the metal decoration produces some small size protuberances on the surface of nanorods. The EDS in Figure 5D is corresponding to that of the $15 \mathrm{~nm} \mathrm{Mn}$ decorated $\mathrm{ZnO}$ nanorods sample. The signal peaks are corresponding to oxygen, zinc, manganese, and copper, respectively. The copper signal in EDS is from the copper grid.

Figure 6 shows the ionization I-V curves of air for a $5 \mathrm{~nm} \mathrm{Mn}$ decorated $\mathrm{ZnO}$ nanorods electrode with and without magnetic field $(6,000 \mathrm{Gs})$. Without a magnetic field, there is no electrical
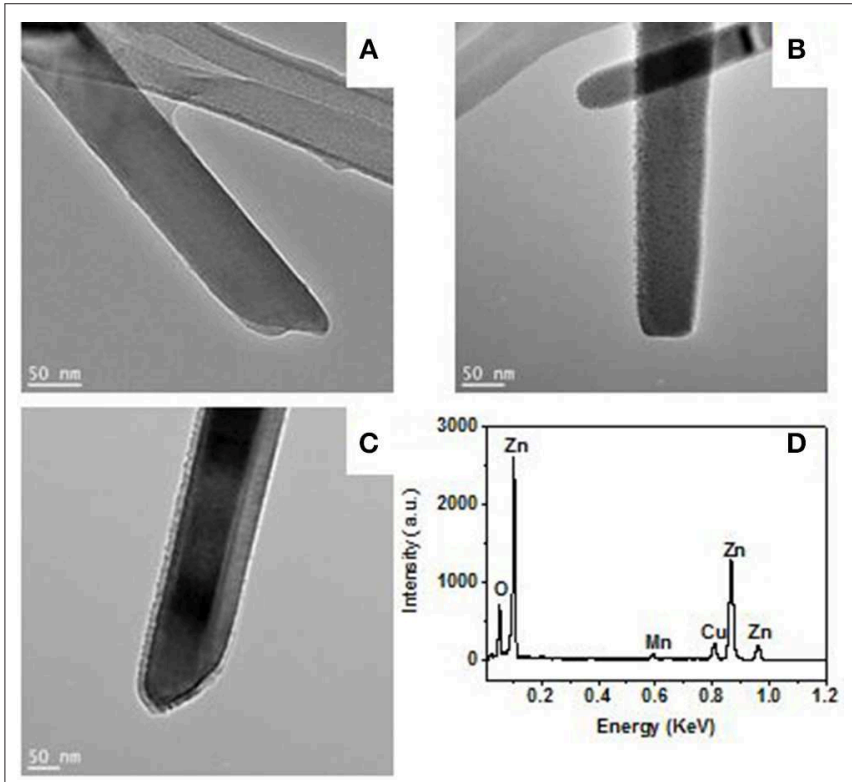

FIGURE 5 | TEM images for a pristine (A), $5 \mathrm{~nm} \mathrm{Mn} \mathrm{(B),} \mathrm{and} 15 \mathrm{~nm} \mathrm{Mn} \mathrm{(C)}$ decorated ZnO nanorod. The EDX (D) is corresponding to $15 \mathrm{~nm} \mathrm{Mn}$ decorated $\mathrm{ZnO}$ nanorod.

breakdown even though the applied voltage increases to $210 \mathrm{~V}$. This means the breakdown voltage of air for $5 \mathrm{~nm}$ Mn decorated $\mathrm{ZnO}$ electrode is higher than $210 \mathrm{~V}$. However, with the magnetic field, the breakdown was observed on the voltage of around $20 \mathrm{~V}$. As shown in Figure 6, there is no obvious difference in the breakdown voltage between parallel or perpendicular magnetic field, thus in the later experiments, the magnetic field is applied perpendicular to the sample plane only. The breakdown voltage of air is about $20 \mathrm{~V}$, which is small enough to be powered by portable batteries and safely handled. Compared to that of the bare $\mathrm{ZnO}$, with $\mathrm{Mn}$ decoration and magnetic field working together, the breakdown voltage of air is decreased more than 10 times. Figure 7 shows the ionization of air for various thickness $\mathrm{Mn}$ decorated $\mathrm{ZnO}$ nanorods under magnetic field. The breakdown voltage of air increases with the thickness of 


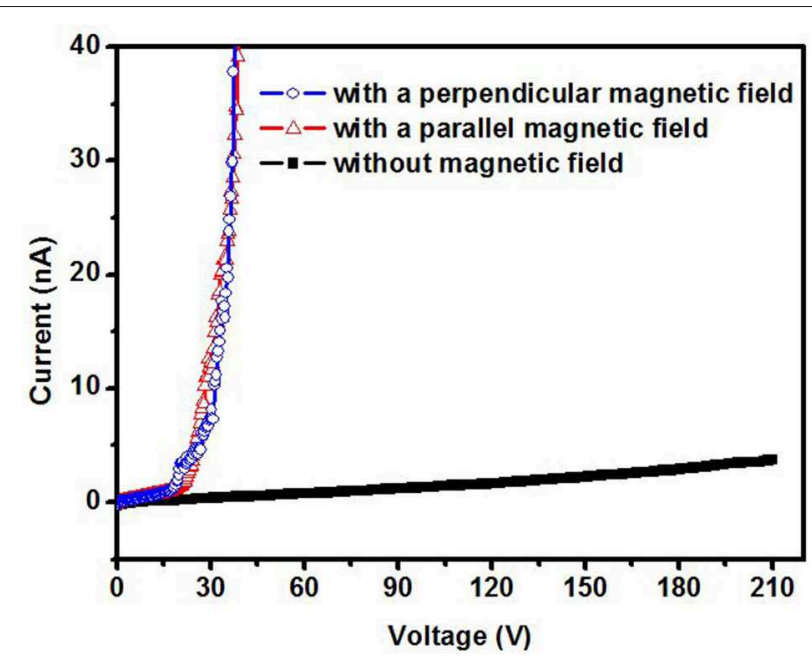

FIGURE 6 | Air ionization curve for a $5 \mathrm{~nm}$ Mn decorated $\mathrm{ZnO}$ nanorods electrode under parallel and perpendicular magnetic field.

decoration increasing. This may be due to that with thickness increasing, the protuberances diameter increases. Figure $\mathbf{8}$ shows that $5 \mathrm{~nm}$ of $\mathrm{Au}, \mathrm{Fe}_{19} \mathrm{Ni}_{81}$ decoration together with a magnetic field can also reduce the breakdown voltage of air. The difference among various metal decorations will be studied in the future. Here is to show that magnetic field combined with various metal decorations is effective to reduce $V_{\mathrm{EB}}$. The role of metal decoration is to produce small size protuberances on the surface of nanorods and then creates a higher electric field at the surface of protuberances (Wang et al., 2011). Besides the change in surface roughness, the surface states created by decoration are also important in reducing the ionization voltage (Karaagac and Islam, 2014). The decoration may unintentionally introduce impurity into the semiconductor and create high density of surface states (Karaagac and Islam, 2014). However, the effect of sole metal decoration on the electrical field enhancement is not enough to decrease $V_{\mathrm{EB}}$ to a safe handle level. A magnetic field is helpful to decrease $V_{\mathrm{EB}}$. The role played by the magnetic field on ionization will be discussed in the following.

In order to confirm that the gas ionization voltage is still the distinct character of gases when the electrode is decorated with metal and putting under a magnetic field, Figure 9 shows the ionization of IPA and air for a $5 \mathrm{~nm} \mathrm{Mn}$ decorated $\mathrm{ZnO}$ electrode under magnetic field. As shown in Figure 9, the breakdown voltage is distinct for IPA and air.

Electron impact ionization (EII) and field ionization (FI) processes were, respectively, proposed for nanomaterial configured as a cathode (Huang et al., 2009; Chivu and Kahrizi, 2013; Mahmood et al., 2013) and an anode (Longwitz et al., 2001; Huang et al., 2009). If the nanorod was biased as a cathode, electrons would be extracted form cathode and in turn be accelerated and impact with gas molecular. Then the gas molecular would be ionized by electron bombardment (Longwitz et al., 2001; Huang et al., 2009; Chivu and Kahrizi, 2013; Mahmood et al., 2013). If the nanorod was biased as an

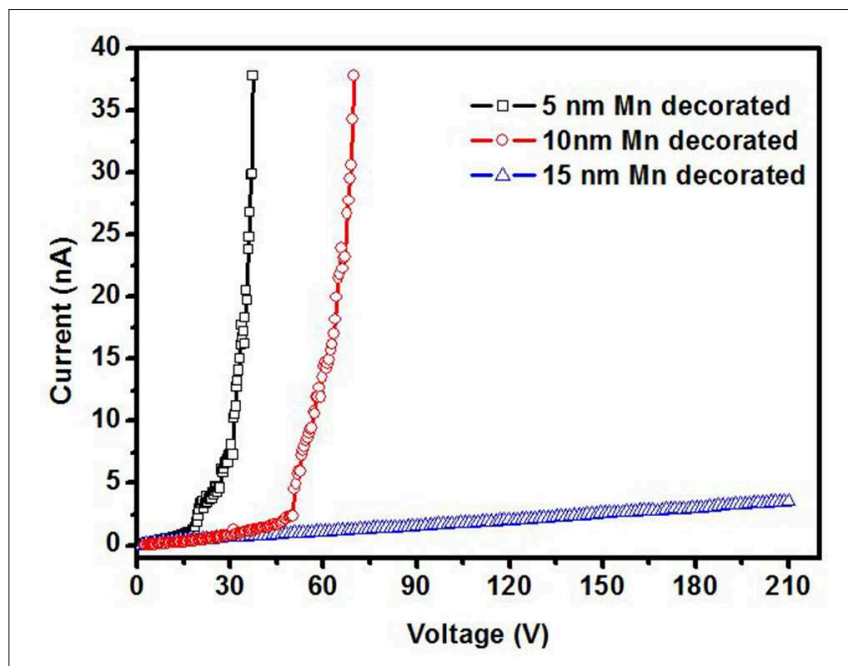

FIGURE 7 | Air ionization for ZnO nanorods electrode decorated with various $\mathrm{Mn}$ thickness under perpendicular magnetic field of 6,000 Gs.

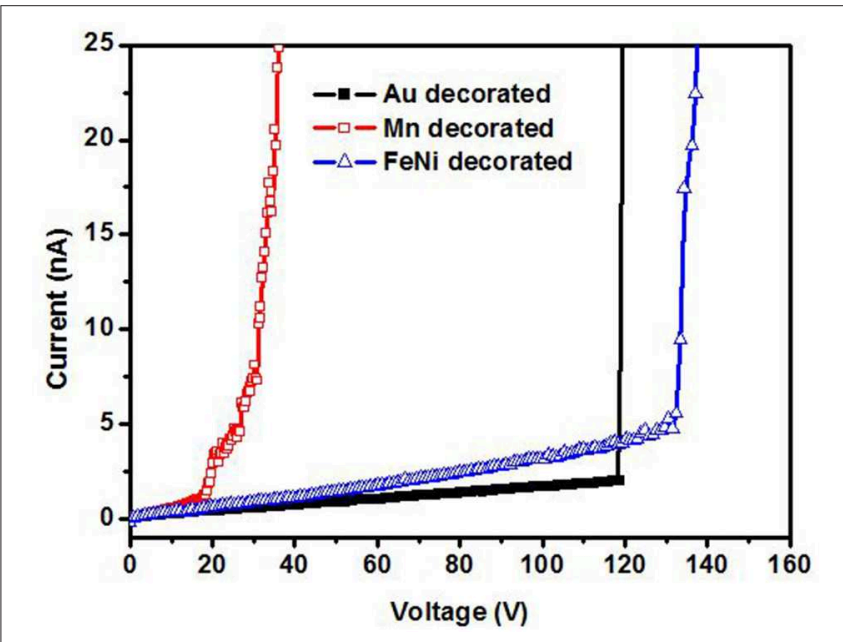

FIGURE 8 | Air ionization for various metal decorated ZnO nanorods under perpendicular magnetic field (6,000 Gs).

anode, gases would be ionized near the surface of nanotips by strong field extraction of the most loosely bound electrons from gas molecules (Longwitz et al., 2001). In our experiment, the $\mathrm{ZnO}$ nanorods were biased as an anode. In the absence of a magnetic field, the ionization of acetone, IPA, and ethanol should be FI. The ionization energy of these organic gases is low enough so that the electrical field is strong enough to extraction electron from these gases molecular and cause gases electrical breakdown. In the case of air, the ionization energy is high, and the electrical field is not strong enough so that no electrical breakdown happens in the applied voltage region. Although there is no gas electrical breakdown, there would be some electrons in the device space such as the cosmic radiation introduced electrons (Azmoodeh et al., 2009) and cathode emission electrons (Huang et al., 2009). Therefore, in 


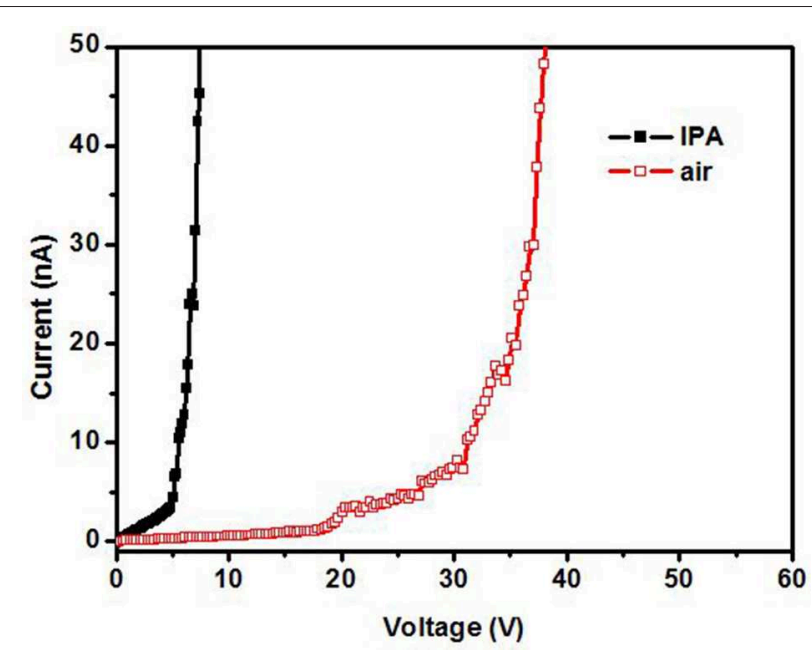

FIGURE 9 | lonization in IPA and air for a $5 \mathrm{~nm} \mathrm{Mn}$ decorated $\mathrm{ZnO}$ nanorods electrode under magnetic field.

the presence of a magnetic field, the efficiency of electron impact ionization and the second electron emission from cathode would be enhanced (Haydon et al., 1971; Li and Uhm, 2004; Petraconi et al., 2004; Radjenović and Radjenović, 2006; Wais et al., 2011). A transverse magnetic field can exert action on the motion of electrons, which makes the gyro-motion of electrons, reduces the mean free path of electrons and increases the number of collision and ionization coefficient ( $\mathrm{Li}$ and Uhm, 2004). The effect of a longitudinal magnetic field on the motion of the electron is to reduce the lateral diffusion of electrons and effectively increases the collision frequency between electrons and the gas particles, thus increasing the ionization efficiency (Petraconi et al., 2004). Therefore, more and more free electrons and ions will be created if the process continues. When the number of free electrons increases exponentially, there will be the flow of a very large current that leads to the breakdown of gases. In a crossed magnetic and electric field, the breakdown voltage is expressed as Radjenović and Radjenović (2006):

$$
V_{E B}=\frac{B_{k}^{k} p d \sqrt{1+C\left(B^{2} / P^{2}\right)}}{\left[\ln A_{k} / \Gamma_{k}+\ln \left(p d \sqrt{1+C\left(B^{2} / P^{2}\right)}\right)\right]^{k}}
$$

Where $p$ is the gas pressure in units of Torr, $k$ has been empirically determined to be one for the molecular gases and two for the atomic gases. $A_{k}$ and $B_{k}$ is the coefficients, for nitrogen $A_{k}=12$

\section{REFERENCES}

Azmoodeh, N., Chivu, N., and Sadeghian, R. B. (2009). "A silver nanowire based gas ionization sensor Eurocon,” in IEEE EUROCON 2009 (St.-Petersburg), 1231.

Baghgar, M., Abdiand, Y., and Arzi, E. (2009). Fabrication of low pressure field ionization gas sensor using bent carbon nanotubes. J. Phys. D Appl. Phys. 42:135502. doi: 10.1088/0022-3727/42/13/135502 $\mathrm{cm}^{-1}$ Torr $^{-1}$ and $B_{k}=342 \mathrm{Vcm}^{-1}$ Torr $^{-1} . B$ is the magnetic flux density expressed in Gauss and $C$ is a constant. $\Gamma_{k}$ is related to the value of yield per ion $\gamma$ as $\Gamma_{k}=\ln \left(1+\frac{1}{\gamma}\right)$. It was confirmed that $V_{\mathrm{EB}}$ could be effectively decreased by the magnetic field (Radjenović and Radjenović, 2006). As seen from Equation (2), the breakdown voltage of gas is indeed dependent on the magnetic field $\mathrm{B}$.

\section{CONCLUSION}

The gas ionization for $\mathrm{ZnO}$ nanorods electrode was explored. It was found that metal $\left(\mathrm{Mn}, \mathrm{Fe}_{19} \mathrm{Ni}_{81}\right.$, and $\left.\mathrm{Au}\right)$ decoration together with a magnetic field can effectively reduce the electrical breakdown voltage of gases. The metal decoration introduces small size protuberances on the surface and thus increase the electric field enhancement. However, this enhancement is not enough to reduce the breakdown voltage of air to a safe voltage. The air electrical breakdown voltage as a function of metal decoration thickness and magnetic field direction was investigated. The magnetic field would increase the efficiency of ionization and second electron emission. Therefore, the electrical breakdown voltage of gases is reduced in the presence of metal decoration and magnetic field significantly.

\section{DATA AVAILABILITY}

All datasets generated for this study are included in the manuscript/supplementary files.

\section{AUTHOR CONTRIBUTIONS}

CL and ZZ conducted the experimental work together, as well contributed to the data analysis and manuscript preparation. JC and HS contributed to sensor measurements and data interpretation. $\mathrm{XX}$ and $\mathrm{XZ}$ contributed to the discussion of experimental results and manuscript preparation. WZ guided the entire experimental process and performed final revision for the manuscript.

\section{FUNDING}

The authors thank to the funding support from Louisiana Board of Regents Nos. LEQSF (2007-12)-ENH-PKSFI-PRS04 and LESQSF (2011-13)-RD-B-08. ZZ, XX, and XZ thank the support of the National Natural Science Foundation of China (U1630126). 
Filip, V., Nicolaescu, D., Tanemura, M., and Okuyama, F. (2001). Modeling the electron field emission from carbon nanotube films. Ultramicroscopy 89, 39-49. doi: 10.1016/S0304-3991(01)00107-3

Haydon, S. C., Mcintosh, A. I., and Simpson, A. A. (1971). The effect of magnetic fields on electron energy distribution functions and derived collision frequencies. J. Phys. D Appl. Phys. 4:1257. doi: 10.1088/0022-3727/4/9/304

Hou, Z. Y., Xu, D., and Cai, B. (2006). Ionization gas sensing in a microelectrode system with carbon nanotubes. Appl. Phys. Lett. 89:213502. doi: 10.1063/1.2392994

Huang, J., Wang, J., Gu, C., Yu, K., Meng, F., and Liu, J. (2009). A novel highly sensitive gas ionization sensor for ammonia detection. Sens. Actuat. A 150, 218-223. doi: 10.1016/j.sna.2009.01.008

Karaagac, H., and Islam, M. S. (2014). Enhanced field ionization enabled by metal induced surface states on semiconductor nanotips. Adv. Funct. Mater. 24:2224. doi: 10.1002/adfm.201303308

Li, D. J., Tang, Y. L., Ao, D. Y., Xiang, X., Wang, S. Y., and Zu, X. T. (2019a). Ultra-highly sensitive and selective $\mathrm{H}_{2} \mathrm{~S}$ gas sensor based on $\mathrm{CuO}$ with sub-ppb detection limit. Int. J. Hydrogen Energy 44, 3985-3992. doi: 10.1016/j.ijhydene.2018.12.083

Li, D. J., Zu, X. T., Ao, D. Y., Tang, Q. B., Fu, Y. Q., Guo, Y. J., et al. (2019b). High humidity enhanced surface acoustic wave (SAW) $\mathrm{H}_{2} \mathrm{~S}$ sensors based on sol-gel CuO films. Sens. Actuat. B Chem. 294, 55-61. doi: 10.1016/j.snb.2019.04.010

Li, S. Z., and Uhm, H. S. (2004). Influence of magnetic field on the electrical breakdown characteristics in cylindrical diode. Phys. Plasmas 11:3443. doi: 10.1063/1.1737743

Li, Y., Xing, Q. F., Yan, Y., and Zhou, W. L. (2010). A large quantity synthesis of $\mathrm{ZnO}$ nanoneedles and their polarity determination. J. Nanosci. Nanotech. 10, 2023-2027. doi: 10.1166/jnn.2010.2129

Liao, L., Lu, H. B., Shuai, M., Li, J. C., Liu, Y. L., Liu, C., et al. (2008). A novel gas sensor based on field ionization from $\mathrm{ZnO}$ nanowires: moderate working voltage and high stability. Nanotechnology 19:175501. doi: 10.1088/0957-4484/19/17/175501

Longwitz, R., Lintel, H. V., Carr, R., Hollenstein, C., and Penaud, P. (2001). "Study of gas ionization schemes for micro devices," in The 11th International Conference on Solid State Sensors and Actuators (Munich).

Mahmood, S., Burhanudin, Z. A., and Salman, A. (2013). Field emission model of carbon nanotubes to simulate gas breakdown in ionization gas sensor. J. Appl. Phys. 113:023302. doi: 10.1063/1.4774073

Min, J., Liang, X., Wang, B., Wang, L., Zhao, Y., Shi, W., et al. (2011). The sensitivity and dynamic response of field ionization gas sensor based on $\mathrm{ZnO}$ nanorods. J. Nanopart. Res. 13:5171. doi: 10.1007/s11051-0110500-2

Modi, A., Koratkar, N., Lass, E., Wei, B., and Ajayan, P. M. (2003). Miniaturized gas ionization sensors using carbon nanotubes. Nature 424, 171-174. doi: $10.1038 /$ nature 01777

Nilsson, L., Groening, O., Emmenegger, C., Kuettel, O., Schaller, E., Schlapbach, L., et al. (2000). Scanning field emission from patterned carbon nanotube films. Appl. Phys. Lett. 76:2071. doi: 10.1063/1.126258

Pan, N., Wang, X., Zhang, K., Hu, H., Xu, B., Li, F., and Hou, J. G. (2005). An approach to control the tip shapes and properties of ZnO nanorods. Nanotechnology 16, 1069-1072. doi: 10.1088/0957-4484/ $16 / 8 / 012$
Pan, N., Xue, H., Yu, M., Cui, X., Wang, X., Hou, J. G., et al. (2010). Tip morphology dependent field emission from $\mathrm{ZnO}$ nanorod arrays. Nanotechnology 21:225707. doi: 10.1088/0957-4484/21/22/225707

Petraconi, G., Maciel, H. S., Pessoa, R. S., Murakami, G., Massi, M., Otani, C., et al. (2004). Longitudinal magnetic field effect on the electrical breakdown in low pressure gases. Braz. J. Phys. 34:1662. doi: 10.1590/S0103-97332004000800028

Radjenović, M. R., and Radjenović, B. (2006). The effect of magnetic field on the electrical breakdown characteristics. J. Phys. D Appl. Phys. 39, 3002-3009. doi: 10.1088/0022-3727/39/14/019

Sadeghian, R. B. (2008). A novel gas sensor based on tunneling field ionization on whisker covered gold nanowires. IEEE Sens. J. 8:161. doi: 10.1109/JSEN.2007.912788

Sadeghian, R. B., and Kahrizi, M. (2007). "A low voltage gas ionization sensor based on sparse gold nanorods," in IEEE Sensors Conference (Atlanta, GA).

Wais, S. I., Mohammed, R. Y., and Yousif, S. O. (2011). Influence of axial magnetic field on the electrical breakdown and secondary electron emission in plane parallel plasma discharge. World Acad. Sci. Eng. Technol. 56:1304. doi: 10.5281 /zenodo. 1333588

Wang, H., Zou, C., Tian, C., Zhou, L., Wang, Z., and Fu, D. (2011). A novel gas ionization sensor using Pd nanoparticle-capped ZnO. Nanoscale Res. Lett. 6:534. doi: 10.1186/1556-276X-6-534

Wang, S. Y., Ma, J. Y., Li, Z. J., Su, H. Q., Alkurd, N. R., Zhou, W. L., et al. (2015). Surface acoustic wave ammonia sensor based on $\mathrm{ZnO} / \mathrm{SiO}$ composite film. J. Hazard. Mater. 285, 368-374. doi: 10.1016/j.jhazmat.2014.12.014

Wang, X. Q., Wang, M., He, P. M., Xu, Y. B., and Li, Z. H. (2004). Model calculation for the field enhacement factor of carbon nanotube. J. Appl. Phys. 96:6752. doi: $10.1063 / 1.1814439$

Xiao, J., Zhang, X., and Zhang, G. (2008). Field emission from zinc oxide nanotowers the role of the top morphology. Nanotechnology 19:295706. doi: 10.1088/0957-4484/19/29/295706

Yao, K., Caruntu, D., Wozny, S., Huang, R., Ikuhara, Y. H., Cao, B. B., et al. (2012). Towards one key to one lock: catalyst modified indium oxide nanoparticle thin film sensor array for selective gas detection. J. Mater. Chem. 22, 7308-7313. doi: 10.1039/c2jm15179k

Zhang, Y., Li, S., Zhang, J. Y., Pan, Z., Min, D., Li, X., et al. (2013). Highperformance gas sensors with temperature measurement. Sci. Rep. 3:1267. doi: $10.1038 /$ srep01267

Zhang, Y., Liu, J., and Li, X. (2001). "Study of gas sensor with carbon nanotube film on the substrate of porous silicon," in IVMC 2001. Proceedings of the 14th International Vacuum Microelectronics Conference (Davis, CA).

Conflict of Interest Statement: The authors declare that the research was conducted in the absence of any commercial or financial relationships that could be construed as a potential conflict of interest.

Copyright (C) 2019 Liu, Zheng, Chen, Su, Xiang, Zu and Zhou. This is an open-access article distributed under the terms of the Creative Commons Attribution License (CC $B Y)$. The use, distribution or reproduction in other forums is permitted, provided the original author(s) and the copyright owner(s) are credited and that the original publication in this journal is cited, in accordance with accepted academic practice. No use, distribution or reproduction is permitted which does not comply with these terms. 